\title{
Some Remarks on Conformal Invariant Theories on Four-Lorentz Manifolds *
}

\author{
Tjoe-hian Go \\ Institut für Theoretische Physik, E, ${ }^{\star}$ RWTH Aachen, Federal Republic of Germany
}

Received June 22, 1974; in revised form November 9, 1974

\begin{abstract}
Contrary to the eleven-parameter group consisting of Poincaré-transformations and dilatations, the group of so-called special conformal transformations can act on the Minkowski space $\mathbb{M}^{4}$ only as a local conformal Lie transformation group.

We show that the universal covering space $\tilde{\mathbb{M}} \stackrel{\text { top }}{\approx} \mathbb{R} \times S^{3}$ of the compactified Minkowski space $\mathbb{M}_{c}^{4} \stackrel{\text { top }}{\approx} S^{1} \times S^{3}$, together with an appropriate metric $\tilde{g}$ on it, form a suitable Lorentz manifold $(\mathbb{M}, \tilde{g})$ that admits universal covering group $\overline{\mathrm{SO}}_{0}(2,4) / Z_{2}$ of the "conformal group" of $\mathbb{M}^{4}$ as a transitive Lie transformation group. This group respects the causality notion on $(\tilde{\mathbb{M}}, \tilde{g})$ usually defined on a Lorentz manifold. However, $(\tilde{\mathbb{M}}, \tilde{g})$ possesses only seven isometries in contrast to the well-known ten isometries on the Minkowski space $\mathbb{M}^{4}$, which correspond to conservation of energy-momentum and angular-momentum.
\end{abstract}

\section{§ 1. Introduction}

Many papers have been written discussing conformal invariant quantum field theories (QFT) (cf. [27, 30] and the references quoted there). Some crucial fundamental questions, especially the compatibility of the group of conformal transformations with the notion of causality as usually defined on a Lorentz manifold (cf. [33], [9]: Chapter VI), are either bypassed by considering another space-time e.g. the Euclidean space-time with definite metric [17], or simply ignored by considering the "group of infinitesimal conformal transformations on Minkowski space $\mathbb{M}^{4}$ " instead of the "group of (finite) conformal transformations on $\mathbb{M}^{4}$ ", which does not exist.

The "Euclidean procedure" would be more satisfactory, if the equivalence of the Wightmann QFT (which is formulated essentially in $\mathbb{M}^{4}$ ) with the Euclidean QFT (cf. [21]) can really be guaranteed without additional assumption [5].

As to the second approach, we want to stress that the meaning of the "group of infinitesimal special conformal transformations on $\mathbb{M}^{4}$ " is somewhat different from the meaning of the group of infinitesimal Poincare transformations on $\mathbb{M}^{4}$. The special conformal transformations can be considered on Minkowski space only as a local group of diffeomorphisms ${ }^{1}$. It can be defined as a global transformation group on compactification of $\mathbb{M}^{4}$ (denoted by $\mathbb{M}_{c}^{4}$ resp. $\hat{\mathbb{M}}_{c}^{4}$ below) $[7,23,25]$, but in that case no global causal structure can be defined. However there is still a local causal structure which can be lifted to a global causal structure if one con-

$\star$ Supported by the Bundesministerium für Forschung und Technologie.

$\star \star$ Present address: Institut für Theor. Physik, University of Bern, Bern, Switzerland.

1 i.e., roughly speaking, each element consists of a group element of the special conformal group together with a domain of definition which is not the whole Minkowski space. Therefore this set is not a group in the usual sense. For an exact definition see (6.5). 
siders the universal covering space $\tilde{\mathbb{M}}$ of compactified Minkowski space. On this space the universal covering group $\mathrm{SU}(2,2)$ of the "conformal group" $\mathrm{SO}_{0}(2,4) / Z_{2}$ of $\mathbb{M}^{4}$ acts as transitive transformation group which conserves the causal ordering and allows a conformal metric $\tilde{g}$ (i.e. metric up to a positive factor) which is invariant under the mentioned transformations. There are some attemps [19, $28,30]$ to establish a well-defined conformal invariant theory that is compatible with causality on $\tilde{\mathbb{M}}$.

Since the space $(\tilde{\mathbb{M}}, \tilde{g})$ has no constant curvature it does not admit ten Killing vector fields. We show that it admits at most seven such fields in contrast to ordinary Minkowski space where we have the ten Killing vector fields corresponding to the conservation of energy-momentum and angular-momentum.

This paper is organized as follows: In $\S 2$, we start with the fourfold covering $\mathrm{SU}(2,2)$ of the "conformal group" of $\mathbb{M}^{4}$. The action of $\widetilde{\mathrm{SU}(2,2)}$ on $\tilde{\mathbb{M}}$ is derived in $\S 3$, where we shall also give the explicit transformation laws of the subgroups $\tilde{D}, \tilde{T}, \tilde{L}, \tilde{C}$ on $\tilde{\mathbb{M}}$. These two sections are based on the papers of Schaaf [26] and Rühl [25]. In $\S 4$, the metric structure of the spaces $\tilde{\mathbb{M}}, \hat{\mathbb{M}}_{c}^{4}$ and $\mathbb{M}^{4}$ are considered. A Lorentz metric $\tilde{g}$ that is compatible with the conformal structure of $\tilde{\mathbb{M}}$ gives rise to the Lorentz manifold $(\tilde{\mathbb{M}}, \tilde{g})$. The isometry groups on $(\tilde{\mathbb{M}}, \tilde{g})$ are investigated. In $\S 5$ we first recall some concepts of causality on Lorentz manifolds that are well-known in general relativity. The conformal invariant causality notion on $\overline{\mathbb{M}}$ is then confirmed. In the last section, we make some remarks about conformal theories formulated on different Lorentz manifolds. In $\S 6.1$. we find out that the reason for the causality violating property of the finite special conformal mapping $c$ on $\mathbb{M}^{4}$ is simply because $c$ is not even a homeomorphism of $\mathbb{M}^{4}$ onto $\mathbb{M}^{4}$. In $\S 6.2$., we make some remarks on the notion of "the group of infinitesimal conformal transformations" on $\mathbb{M}^{4}$. In the last $\S$ 6.3., after some discussions, the question concerning the usefulness of $(\tilde{\mathbb{M}}, \tilde{g})$ as a physically meaningful framework for a conformally invariant theory is considered, cf. [16].

Some notations used in this paper come from certain texbooks $[14,31]$. We denote the vector space of real $n$-tuples $x=\left(x^{1}, \ldots, x^{n}\right)$ with the bilinear form

$$
b_{s}^{n}(x, y):=-\sum_{i=1}^{s} x^{i} y^{i}+\sum_{j=s+1}^{n} x^{j} y^{j}, \quad 0 \leqq s \leqq n,
$$

by $\mathbb{R}_{s}^{n}$. $\mathbb{R}_{s}^{n}$ (or the space isomorphic to it) then has signature $(s, n-s)$. By a Lorentz metric in a four dimensional manifold we mean a metric of signature $(3,1)$. The summation convention shall be used throughout the paper. The indices $\{p, q, r \ldots\}$ shall run from 1 to $4 ;\{i, j, k \ldots\}$ shall run from 1 to $3 ;\{\mu, v, \ldots\}$ from 0 to 4 or 5 . The subscript "0" in $\mathrm{SO}_{0}(2,4)$ shall denote the identity component of the group.

\section{$\S$ 2. The Universal Covering Group $\overline{\mathrm{SU}(2,2)}$ of Conformal Group $\mathrm{SU}(2,2)$ and Its Subgroups $\tilde{T}, \tilde{C}, \tilde{D}, \tilde{L}$}

\subsection{The Group $\mathrm{SU}(2,2)$ and the Universal Covering Group $\widetilde{\mathrm{SU}(2,2)}$}

The group $\mathrm{SU}(2,2)$ consists of the complex unimodular $4 \times 4$-matrix $H$ satisfying

$$
H^{+} G H=G, G=\left(\begin{array}{ll}
-\mathrm{E} & \\
& E
\end{array}\right), \operatorname{det} H=+1,
$$


where $E:=\left(\begin{array}{ll}1 & 0 \\ 0 & 1\end{array}\right)$ is a $2 \times 2$-unit matrix. Using $2 \times 2$-blocks $A, B, C, D, H$ can be written as $H=\left(\begin{array}{ll}A & B \\ C & D\end{array}\right)$. The constraint (2.1) leads to the unique polar decomposition of Graev $[8,26]$

$$
H=\left(\begin{array}{cc}
N_{1} & z N_{2} \\
z^{+} N_{1} & N_{2}
\end{array}\right)\left(\begin{array}{ll}
u_{1} & \\
& u_{2}
\end{array}\right)\left(\begin{array}{cc}
\Omega_{1}(x) & \\
& \Omega_{2}(x)
\end{array}\right)=: Z U \Omega(x),
$$

where $z:=B \cdot D^{-1}$ belongs to the generalized unit disc

$$
D(2,2):=\left\{z \mid 0 \leqq z z^{+}<E\right\}=\left\{z \mid 0 \leqq z^{+} z<E\right\} ;
$$

$N_{1}:=\left(1-z z^{+}\right)^{-1 / 2}, N_{2}:=\left(1-z^{+} z\right)^{-1 / 2}$ are positive definite $2 \times 2$-matrices; $u_{i} \in \mathrm{SU}(2), i=1,2 ; \Omega_{1}(x)=\Omega_{2}^{+}(x)=\left(\begin{array}{ll}e^{i x} & \\ & \\ & 1\end{array}\right) ; Z$ is a positive definite Hermitian

The group law $H H^{\prime}=H^{\prime \prime}$ of $\mathrm{SU}(2,2)$ in the above parametrization $H=Z U \Omega$ reads

$$
Z U \Omega \circ Z^{\prime} U^{\prime} \Omega^{\prime}=Z^{\prime \prime} U^{\prime \prime} \Omega^{\prime \prime},
$$

where the explicit forms of $Z^{\prime \prime}, U^{\prime \prime}$ and $\Omega^{\prime \prime}$ can be found in [26], § 2. (2.2) implys that $D(2,2)$ can be identified with the homogeneous space $\operatorname{SU}(2,2) / S(U(2) \otimes U(2))$. Denoting the center of SU(2,2) by $Z_{4}:=\left\{\left(\begin{array}{ll}A & O \\ O & A\end{array}\right), A=e^{2 i n / 4} E, n=0,1,2,3\right\}$, the group element $H \in \mathrm{SU}(2,2) / Z_{4}$ acts as the automorphism of $D(2,2)$ by

$$
\begin{gathered}
H=Z U \Omega=\left(\begin{array}{ll}
A & B \\
O & D
\end{array}\right): D(2,2) \rightarrow D(2,2) \\
z^{\prime} \mapsto \hat{z}\left(z, z^{\prime}\right):=\left(A z^{\prime}+B\right)\left(C z^{\prime}+D\right)^{-1} .
\end{gathered}
$$

The seven-dimensional boundary $\partial D(2,2)$ contains the so-called Shilov boundary $\mathbb{S}[25]$, which is a four-dimensional subset of $\partial D(2,2)$ consisting of all unitary $2 \times 2$-complex matrix $s$ :

$$
\mathbb{S}:=\left\{s \in \partial D(2,2) \mid s^{+}=s^{-1}\right\} .
$$

By analyticity, the invariant action of $\mathrm{SU}(2,2) / Z_{4}$ on $\mathrm{S}$ is also given by (2.5).

The element $\tilde{H}$ of the universal covering group $\widetilde{\operatorname{SU}(2,2)}$ is of the form $(Z, U, x)$, where $x \in \mathbb{R}$ and $Z, U$ are given above. The group composition law of $\operatorname{SU}(2,2)$ is given in [26] by

$$
(Z, U, x) \circ\left(Z^{\prime}, U^{\prime}, x^{\prime}\right):=\left(Z^{\prime \prime}, U^{\prime \prime}, x^{\prime \prime}:=x+x^{\prime}+F\left(Z, Z_{u \Omega(x)}^{\prime}\right)\right),
$$

where $Z^{\prime \prime}, U^{\prime \prime}$ are given in (2.4) and

$$
\begin{aligned}
F: D(2,2) \times D(2,2) \rightarrow \mathbb{R}, F\left(Z, Z^{\prime}\right): & =f\left(z z^{+}\right)=-f\left(z^{+} z^{\prime}\right) \\
: & =\operatorname{Im} \operatorname{tr} \ln \left(E+z z^{\prime+}\right)
\end{aligned}
$$

is of class $C^{\infty}$ with range in the open intervall $(-\pi,+\pi)$. Furthermore, we have

$$
e^{i f\left(z z^{\prime+}\right)}=\operatorname{det}\left(E+z z^{\prime+}\right) /\left|\operatorname{det}\left(E+z z^{+}\right)\right|=: \omega\left(z z^{+}\right)
$$

and

$$
\operatorname{det} \hat{z}(z, u)=\left(\omega\left(z u^{+}\right)\right)^{2} \operatorname{det} u, \quad \text { for } \quad u \in U(2) \text {. }
$$




\subsection{The Subgroups $\tilde{D}, \tilde{L}, \tilde{T}, \tilde{C}$ of $\widetilde{\mathrm{SU}(2,2)}$}

Using the results of [25], we can readily write down in $(A, B, C, D)$-language the various subgroups of $\operatorname{SU}(2,2)$ corresponding to

dilatation:

$$
\hat{D}=\left(\begin{array}{cc}
\mathrm{CH} \varrho E & -\mathrm{SH} \varrho E \\
-\mathrm{Sh} \varrho E & \mathrm{Ch} \varrho E
\end{array}\right) ;
$$

$$
\mathrm{SL}(2, \mathbb{C}) / Z_{2}: \quad \hat{L}=\left(\begin{array}{ll}
A & B \\
C & D
\end{array}\right), \quad \begin{aligned}
& A=D=a_{R}^{0} \sigma_{0}-i a_{I}^{k} \sigma_{k} \\
& B=C=-i a_{I}^{0} \sigma_{0}+a_{R}^{k} \sigma_{k}
\end{aligned}
$$

where

$$
a^{\mu}=g^{\mu v} a_{v}=a_{R}^{\mu}+i a_{I}^{\mu} \in \mathbb{C}, \quad\left(g^{\mu v}\right)=\left(g_{\mu v}\right)=\left(\begin{array}{llll}
1 & & & \\
& -1 & & \\
& -1 & \\
& & & -1
\end{array}\right)
$$

$\operatorname{det} a_{\mu} \sigma_{\mu}=+1$ and $\sigma_{k}$ are the usual Pauli matrices;

translation: $\quad \hat{T}=\left(\begin{array}{cc}E+i T / 2 & i T / 2 \\ -i T / 2 & E-i T / 2\end{array}\right), \quad T=t_{\mu} \sigma_{\mu}, \quad\left(t_{\mu}\right) \in \mathbb{R}^{4} ;$

and special conformal transformation:

$$
\hat{C}=\left(\begin{array}{cc}
E-i C / 2 & i C / 2 \\
-i C / 2 & E+i C / 2
\end{array}\right), \quad C=c_{\mu} \sigma_{\mu}, \quad\left(c_{\mu}\right) \in \mathbb{R}^{4} .
$$

The corresponding universal covering subgroups $\tilde{D}, \tilde{L}, \tilde{T}, \tilde{C}$ can easily be obtained: we merely have to translate the $(A, B, C, D)$-language into $(Z, U, x)$-language by computing $z=B \cdot D^{-1}, N_{1}=\left(E-z^{+} z\right)^{-1 / 2}$ etc. We shall not give the explicit forms of them here.

\section{§ 3. The Action of $\overline{\mathrm{SU}(2,2)}$ on the Universal Covering Space $\tilde{\mathrm{S}}$ of Compactified Minkowski Space $\mathbb{S}$}

\subsection{The Transformation Law and Some Group Properties of $\widetilde{\mathrm{SU}(2,2)}$} as Lie Transformation Group on $\widetilde{\mathbb{S}}$

It is clear that the universal covering space of $\mathbb{S} \equiv U(2)$ is $\mathrm{SU}(2) \times \mathbb{R} \stackrel{\Phi \times \text { id }}{=} S^{3} \times \mathbb{R}$, where the homeomorphism $\Phi$ is given by

$$
\Phi: S^{3} \stackrel{\text { onta }}{\longrightarrow} \mathrm{SU}(2), \quad n=\left(n^{1}, n^{2}, n^{3}, n^{4}\right) \mapsto u(n):=n^{4} \sigma_{0}+i n^{k} \sigma_{k} .
$$

SU(2) inherites the natural $C^{\infty}$-structure of $S^{3}$ via $\Phi$. With respect to which, $\Phi$ is a diffeomorphism. We shall denote the $C^{\infty}$-manifolds $\mathrm{SU}(2) \times \mathbb{R}$ and $S^{3} \times \mathbb{R}$ by the same symbol $\tilde{\mathbb{S}}$. Likewise, $\mathbb{S}$ shall also inherite in the standard unique way (cf. [31] Lemma 1.8.23iii) the $C^{\infty}$-structure of $\tilde{\mathbb{S}}$ via the local homeomorphic covering map

$$
\tilde{p}: \tilde{\mathbb{S}} \rightarrow \mathbb{S}, \quad \tilde{s} \equiv(u(n), \tau) \mapsto u(n) e^{i \tau}
$$

so that $\tilde{p}$ becomes a local diffeomorphism. 
Our task is to define a mapping $\tilde{h} \in \widetilde{\mathrm{SU}(2,2)}: \tilde{\mathbb{S}} \rightarrow \tilde{\mathbb{S}}$ such that it not only guarantees the commutativity of the following diagram

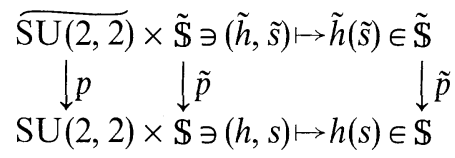

where $h(s)$ is given in (2.5), $\tilde{p}$ by (3.2) and $p$ by

$$
p: \widetilde{\mathrm{SU}(2,2)} \rightarrow \mathrm{SU}(2,2), \quad(Z, U, x) \mapsto Z U \Omega(x),
$$

but also satisfies the conditions of being a Lie transformation group of $\tilde{\mathbb{S}}$.

Let $s \in \mathbb{S}$ with det $s=e^{2 i \tau}$, then $\tilde{s}=\left(s e^{-i \tau}, \tau\right) \in \tilde{\mathbb{S}}$. We define $\tilde{h}(\tilde{s})$ as follows:

$$
\begin{aligned}
\tilde{h} & =(Z, U, x) \in \widetilde{\mathrm{SU}(2,2)}: \tilde{\mathbb{S}} \rightarrow \tilde{\mathbb{S}}, \quad \tilde{s}=\left(s e^{-i \tau}, \tau\right) \mapsto \tilde{h}(\tilde{s}) \\
\tilde{h}(\tilde{s}): & =\left(\left(\hat{z}\left(z, s_{u \Omega(x)}\right) \omega\left(z s_{u \Omega}^{+}\right) e^{-i(x+\tau)}, x+\tau+f\left(z s_{u \Omega(x)}^{+}\right)\right)\right) \\
& =:\left(s^{\prime}\left(\operatorname{det} s^{\prime}\right)^{-1 / 2}, x+\tau+f\left(z s_{u \Omega(x)}^{+}\right)\right)
\end{aligned}
$$

where $\hat{z}, \omega, f$ are given in (2.5), (2.9) and (2.8) respectively. By means of (2.10), (3.1) and (3.5), the action of the subgroups $\tilde{D}, \tilde{L}, \tilde{T}, \tilde{C}$ of $\widetilde{\mathrm{SU}(2,2)}$ on $\tilde{\mathbb{S}}$ can be computed.

Let $h=p(\tilde{h})=Z U \Omega(x)=\left(\begin{array}{ll}A & B \\ C & D\end{array}\right) \in \mathrm{SU}(2,2)$ corresponds to $\tilde{h} \in \widetilde{\mathrm{SU}(2,2)}$. We rewrite the action of $\mathrm{SU}(2,2)$ on $\mathbb{S}$ given in $(2.5)$ by

$$
\begin{aligned}
h: \mathbb{S} \rightarrow \mathbb{S}, \quad s=u(n) e^{i \tau} \mapsto s_{h} & =(A s+B)(C s+D)^{-1} \\
& =: e^{i \tau}\left(K_{h}^{4} \sigma_{0}+i \vec{K}_{h} \cdot \vec{\sigma}\right) / F_{h}(s)
\end{aligned}
$$

where $F_{h}(s):=\operatorname{det}(C s+D)$. Introducing $N_{h}:=\left[\left(K_{h}^{4}\right)^{2}+\vec{K}_{h}^{2}\right]^{1 / 2}$ we thus obtain $\left(\operatorname{det} s_{h}\right)^{1 / 2}=e^{i \tau} F_{h}^{-1} N_{h}$.

Rewriting (3.5) also for $\tilde{\mathbb{S}}$,

$$
\tilde{h}: \tilde{\mathbb{S}} \rightarrow \tilde{\mathbb{S}}, \quad(n, \tau) \mapsto\left(n^{\prime}, x+\tau+f\left(z\left(u(n) e^{i \tau}\right)_{u \Omega(x)}^{+}\right)\right),
$$

it then follows that $n^{\prime p}=K_{h}^{p} N_{h}^{-1} \in S^{3}, p=1, \ldots, 4$, while for $\tilde{h} \in \widetilde{\operatorname{SU}(2,2)}, f\left(\zeta_{\tilde{h}}\right)$, $\zeta_{\tilde{h}}:=z(\tilde{h})\left(u(n) e^{i \tau}\right)_{u \Omega(x)}^{+}$, is computable with the help of its equivalent form $f\left(z z^{\prime+}\right)$ $=\operatorname{Im} \Sigma_{i=1}^{2} \ln \left(E+\lambda_{i} E\right)$, where $\left\{\lambda_{i}\right\}$ are eigenvalues of $z z^{\prime+}$.

We give the results of our computations:

The eigenvalues $\lambda_{1,2}^{\tilde{D}}$ for $\zeta_{\tilde{D}}$ are

$$
\lambda_{1,2}^{\tilde{D}}=-\operatorname{th} \varrho e^{-i \tau}\left(n^{4} \pm i|\vec{n}|\right) ;
$$

$f\left(\zeta_{\tilde{D}}\right)$ can therefore be given explicitely as a series

$$
f\left(\zeta_{\tilde{D}}\right)=\sum_{\nu=1}^{\infty}(\operatorname{th} \varrho)^{v} \frac{\sin \tau \nu}{v}\left[\left(n^{4}+i|\vec{n}|\right)^{v}+\left(n^{4}-i|\vec{n}|\right)^{v}\right] .
$$

The same can also be done for $\tilde{L}$-subgroup, because the expression within the square-root in the eigenvalues of $\zeta_{\tilde{L}}$

$$
\begin{aligned}
\lambda_{1,2}^{\tilde{L}} & =e^{-i \tau}\left[-\vec{J}^{\prime} \cdot \vec{n} \pm \sqrt{\vec{J}^{2}-\left(\vec{J}^{\prime} \cdot \vec{n}\right)^{2}}\right], \\
\vec{J}: & =\left(a_{R}^{0} \vec{a}_{R}+a_{I}^{0} \vec{a}_{I}-\vec{a}_{R} \times \vec{a}_{I}\right) / \operatorname{det} A, \\
\vec{J}^{\prime} & :=\vec{J}+\left(2 \vec{a}_{R} \times \vec{a}_{I} / \operatorname{det} A\right), \quad \operatorname{det} A:=\left(a_{R}^{0}\right)^{2}+\vec{a}_{I}^{2}
\end{aligned}
$$


is semi-positive definite that can be seen by means of the homomorphism $\Lambda$ mapping $\operatorname{SL}(2, \mathbb{C})$ onto $L_{+}^{\uparrow}$. The more practical form of $f\left(\zeta_{\tilde{L}}\right)$ is however

$$
f\left(\zeta_{\tilde{L}}\right)=\operatorname{Im} \ln \left(1-2 i e^{-i \tau} \vec{J}^{\prime} \cdot \vec{n}-e^{-2 i \tau} \vec{J}^{2} / 4\right) .
$$

We note that it does not depend on $n^{4}$-variable. Likewise,

$f\left(\zeta_{\tilde{T}}\right)=\operatorname{Im} \ln \left\{1+i \frac{e^{-i \tau}}{\left|R_{2}\right|^{2}}\left(n^{4}\left(\left(R_{2}+i R_{1}\right) R_{1}-i \overrightarrow{t^{2}}\right)+i R_{2} \vec{t} \cdot \vec{n}\right)-\frac{e^{-2 i \tau}}{4\left|R_{2}\right|^{2}}(t \cdot t) R_{2}\right\}$

$f\left(\zeta_{\tilde{c}}\right)=\operatorname{Im} \ln \left\{1+i \frac{e^{-i \tau}}{\left|R_{2}^{\prime}\right|^{2}}\left(n^{4}\left(\left(R_{2}^{\prime}-i R_{1}^{\prime}\right) R_{1}^{\prime}+i \vec{c}^{2}\right)+i R_{2}^{\prime} \vec{c} \cdot \vec{n}\right)-\frac{e^{-2 i \tau}}{4\left|R_{2}^{\prime}\right|^{2}}(c \cdot c) R_{2}^{\prime}\right\}$ where

$$
\begin{aligned}
& R_{1}:=t^{0}-i \frac{t \cdot t}{2}, \quad R_{2}:=1-i t^{0}-\frac{t \cdot t}{2} \\
& R_{1}^{\prime}:=c^{0}+i \frac{c \cdot c}{2}, \quad R_{2}^{\prime}:=1+i c^{0}-\frac{c \cdot c}{2} .
\end{aligned}
$$

$n^{\prime p}=K_{h}^{p} N_{h}^{-1}$ are given by

$$
\begin{aligned}
& \left\{\begin{array}{l}
K_{D}^{4}=n^{4}\left(\mathrm{Sh}^{2} \varrho+\mathrm{Ch}^{2} \varrho\right)-\left(e^{i \tau}+e^{-i \tau}\right) \text { Sh } \varrho \mathrm{Ch} \varrho \\
\vec{K}_{D}=\vec{n}
\end{array}\right. \\
& \left\{\begin{aligned}
K_{L}^{4}= & n^{4} \\
\vec{K}_{L}= & i\left(e^{i \tau}-e^{-i \tau}\right) \operatorname{det} A \cdot \vec{J}+\vec{n}\left(a_{R} \cdot a_{R}+a_{I} \cdot a_{I}\right) \\
& +2 \vec{a}_{I}\left(\vec{a}_{I} \cdot \vec{n}\right)+2 \vec{a}_{R}\left(\vec{a}_{R} \cdot \vec{n}\right)+2 a_{R}^{0}\left(\vec{a}_{I} \times \vec{n}\right)+2 a_{I}^{0}\left(\vec{n} \times \vec{a}_{R}\right)
\end{aligned}\right. \\
& \left\{\begin{array}{l}
K_{T}^{4}=\left(e^{i \tau}+e^{-i \tau}\right) \frac{t \cdot t}{4}-i\left(e^{i \tau}-e^{-i \tau}\right) \frac{t^{0}}{2}+n^{4}\left(1+\frac{t \cdot t}{2}\right)+\vec{t} \cdot \vec{n} \\
\vec{K}_{T}=-\left(e^{i \tau}+e^{-i \tau}\right) \frac{\vec{t}}{2}+\vec{n}-n^{4} \vec{t}
\end{array}\right. \\
& \left\{\begin{array}{l}
K_{C}^{4}=\left(e^{i \tau}+e^{-i \tau}\right) \frac{-c \cdot c}{4}-i\left(e^{i \tau}-e^{-i \tau}\right) \frac{c^{0}}{2}+n^{4}\left(1+\frac{c \cdot c}{2}\right)-\vec{c} \cdot \vec{n} \\
\vec{K}_{C}=-\left(e^{i \tau}+e^{-i \tau}\right) \frac{\vec{c}}{2}+\vec{n}+n^{4} \vec{c} .
\end{array}\right.
\end{aligned}
$$

(3.8) and (3.9) are the explicit group transformation law of $\widetilde{\mathrm{SU}(2,2)}$ on $\tilde{\mathrm{S}}$.

The center $Z_{c}$ of $\widetilde{\mathrm{SU}(2,2)}$, consisting e.g. of elements of the form,

$$
\left(\left(\begin{array}{ll}
E & 0 \\
0 & E
\end{array}\right),\left(\begin{array}{ll}
E & 0 \\
0 & E
\end{array}\right), 2 \pi n\right), \quad n \in Z,
$$

is infinite (cf. [11] p. 239). It is obviously sufficient to guarantee the transitivity of $\widetilde{\mathrm{SU}(2,2)}$ on $\tilde{\mathrm{S}}$. $\overline{\mathrm{SU}(2,2)}$ does not act freely on $\tilde{\mathbb{S}}^{2}$ since there is a subgroup $\{e\} \times \operatorname{Spin}(3) \subset \widetilde{\mathrm{SO}_{0}(2) \times \mathrm{SO}_{0}(4)}$ of $\overline{\mathrm{SU}(2,2)}$ (cf. [11] p. 347) that leaves a point $(\tau, n)$ of $\tilde{\mathbb{S}}$ invariant. $\tilde{\mathrm{SU}}(2,2) / Z_{c} \stackrel{\left({ }^{3.4}\right)}{\approx} \mathrm{SU}(2,2)$ acts effectively on $\tilde{\mathbb{S}}$ which can be

\footnotetext{
2 The author is indebted to Dr. D. Mayer for discussing this point.
} 
seen from the existence of the isotropy subgroup of the point $\left(\tau, n^{1}, n^{2}, n^{3}, n^{4}\right)$ $=(2 \pi, 0,0,0,1) \in \tilde{\mathbb{S}}$, which is given by the 11 -parameter group

$$
[(L \otimes D) \otimes C] / Z_{c} .
$$

It is isomorphic to the isotropy group of the corresponding point $[\eta]=\left[\left(\eta^{0}, \eta^{1}\right.\right.$, $\left.\left.\ldots, \eta^{4}, \eta^{5}\right)\right]=[(0, \ldots, 0,1,1)]$ in $\mathbb{M}_{c}^{4}$ (cf. $\S 3.2$. below),

$$
(L \otimes D) \otimes C \text {. }
$$

That $\widetilde{\mathrm{SU}(2,2)}$ does not act linearly on $\tilde{\mathbb{S}}$ depends on the fact that $\tilde{\mathbb{S}} \stackrel{\text { top }}{\approx} \mathbb{R} \times S^{3}$ is not a flat Euclidean space.

\section{§ 3.2. The Connection of the Transformation Law of $\widetilde{\mathrm{SU}(2,2)}$ on $\tilde{\mathrm{S}}$} with the Transformation Law of $\mathrm{SO}_{0}(2,4)$ on $\hat{\mathbb{M}}_{c}^{4}$

To see the connection of the transformation law of $\overline{\mathrm{SU}(2,2)}$ on $\tilde{\mathbb{S}}$ with the well-known transformation law of conformal group $\mathrm{SO}_{0}(2,4)$ on e.g. $\mathbb{M}_{c}^{4} \stackrel{\text { top }}{\approx} S^{1} \times S^{3}$, let us consider the following homeomorphism

$$
\begin{aligned}
\psi: \mathbb{S} \rightarrow \hat{\mathbb{M}}_{c}^{4}: & =\mathbb{Q}_{0}^{5} / \mathbb{R}_{+}, \\
e^{i \tau} u(n) \mapsto[\eta] & \equiv\left[\left(\eta^{0}, \vec{\eta}, \eta^{4}, \eta^{5}\right)\right]:=\left[\left(\sin \tau, \vec{n}, n^{4}, \cos \tau\right)\right]
\end{aligned}
$$

where $\mathbb{Q}_{0}^{5}$ is the isotropic quadric in the space $\mathbb{R}_{4}^{6} \backslash\{0\}$

$$
\mathbb{Q}_{0}^{5}:=\left\{\eta \in \mathbb{R}_{4}^{6} \backslash\{0\} \mid b_{4}^{6}(\eta, \eta)=0\right\},
$$

and $\mathbb{R}_{+}$is the equivalent relation

$$
\eta \stackrel{\mathbb{R}_{+}}{\sim} \eta^{\prime} \quad \text { iff } \quad \eta^{\prime}=\varrho \eta, \quad \varrho \in \mathbb{R}_{+} .
$$

As before, we have equipped $\mathbb{M}_{c}^{4}$ with the $\psi$-induced $C^{\infty}$-structure of $\mathbb{S}$ to obtain a $C^{\infty}$-manifold $\hat{\mathbb{M}}_{c}^{4}{ }^{3}$

The induced action of $\operatorname{SU}(2,2)$ on $\hat{\mathbb{M}}_{c}^{4}$ is then given by

$$
[\eta]=\left[\left(\sin \tau, \vec{n}, n^{4}, \cos \tau\right)\right] \mapsto\left[\eta^{\prime}\right]=\left[\left(\sin \tau^{\prime}, \vec{n}^{\prime}, n^{\prime 4}, \cos \tau^{\prime}\right)\right]
$$

where $n^{\prime}$ are given in (3.9) and

$$
\left(\eta^{\prime 0}, \eta^{\prime 5}\right)=(\operatorname{Re}, \operatorname{Im}) e^{i \tau} F_{h}^{-1} N_{h}=\left(\sin \tau^{\prime}, \cos \tau^{\prime}\right) .
$$

One readily verify that the usual transformation law of $\mathrm{SO}_{0}(2,4)$ on a six-dimensional space (cf. e.g. [12]) up to a multiplicative factor $N_{h}^{-1}$ would be obtained, if we do not consider the point $\eta$ as a representative of the equivalence class $[\eta]$. The appearance of $N_{h}^{-1}$ is clearly necessary to warrant that the mapping does take place from $S^{1} \times S^{3}$ onto $S^{1} \times S^{3}$.

${ }^{3}$ There is still another compactification of $\mathbb{M}^{4}$, i.e. $\mathbb{M}_{c}^{4}:=\mathbb{Q}_{0}^{5} / \mathbb{R}_{0}$, where the equivalent relation $\mathbb{R}_{0}$ is given by $\eta \stackrel{\mathbb{R}_{\rho}}{\sim} \eta^{\prime}$ iff $\eta^{\prime}=\varrho \eta, \eta \in \mathbb{R} \backslash\{0\}[7] . \mathbb{M}_{c}^{4}$ is therefore twofold covered by $\hat{\mathbb{M}}_{c}^{4}$. The difference of $\mathbb{M}_{c}^{4} \stackrel{\text { top }}{\approx} S^{1} \times S^{3} / Z_{2}$ and $\mathbb{\mathbb { M }}_{c}^{4}$ can at best be seen from their different first fundamental group: $\pi_{1}\left(\hat{\mathbb{M}}_{c}^{4}\right)$ $\cong Z, \pi_{1}\left(\mathbb{M}_{c}^{4}\right) \cong Z \times Z_{2}$, where the group $Z \times Z_{2}$ has a nonabelian group structure, since $Z_{2}$ acts as inversion relative to origin, while $Z$ acts as translation [3]. We shall only consider $\hat{\mathbb{M}}_{c}^{4}$ in the sequel. 


\section{§4. The Universal Covering Space $\mathbb{I}$ of the Conformally Compactified Minkowski Space $\hat{\mathbb{M}}_{c}^{4}$}

4.1. The Space $\tilde{\mathbb{M}}$ and Its Relation to $\hat{\mathbb{M}}_{c}^{4}$ as Well as $\mathbb{M}^{4}$

To get a space corresponding to $\tilde{\mathbb{S}}$ which is on the same footing as the diffeomorphic image of $\mathbb{S}$, i.e. $\widehat{\mathbb{M}}_{c}^{4}$, let us consider the bijective mapping

where

$$
\gamma: \tilde{\mathbb{S}} \rightarrow \mathbb{H}_{3}^{4}, \quad\left(\tau, n^{p}\right) \mapsto y:=\left(\tau, n^{p} \sqrt{\alpha^{2}+\tau^{2}}\right),
$$

$$
\mathbb{H}_{3}^{4}:=\left\{y \in \mathbb{R}_{4}^{5} \mid b_{4}^{5}(y, y)=-\alpha^{2}, \alpha=\text { const }>0\right\} \stackrel{\text { top }}{\approx} \mathbb{R} \times S^{3}
$$

is a hyperbolic subset of $\mathbb{R}_{4}^{5}$. We provide $\mathbb{H}_{3}^{4}$ as usual with the $\gamma$-induced $C^{\infty}$ structure of $\tilde{\mathbb{S}} \cdot \gamma$ then becomes a diffeomorphism while $\mathbb{H}_{3}^{4}$ turns out to be a paracompact regular submanifold of $\mathbb{R}_{4}^{5}$ that shall be denoted by $\tilde{\mathbb{M}}$ in the sequel.

According to the discussions above, the local diffeomorphic covering map $p_{1}: \widetilde{\mathbb{M}} \rightarrow \hat{\mathbb{M}}_{c}^{4}$ is given uniquely by

$$
p_{1}: \tilde{\mathbb{M}} \rightarrow \hat{\mathbb{M}}_{c}^{4}, \quad\left(\tau, n^{p} \sqrt{\alpha^{2}+\tau^{2}}\right) \mapsto[\eta]:=\left[\left(\sin \tau, n^{p}, \cos \tau\right)\right] .
$$

In contrast, the Minkowski space $\mathbb{M}^{4}$ is identified in [7] merely as a homeomorphic image of a local chart $\left(U_{\varkappa}, \varphi_{\varkappa}\right)$ of $\mathbb{M}_{c}^{4}$,

$$
\varphi_{\varkappa}: U_{\varkappa} \rightarrow \mathbb{R}^{4} \approx \varphi_{\varkappa}\left(U_{\varkappa}\right), \quad[\eta] \mapsto\left(x^{\mu}\right):=\left(\eta^{\mu} / \varkappa\right), \quad \varkappa:=\eta^{4}+\eta^{5} \neq 0,
$$

where the domain of $\varphi_{\varkappa}$ is an open dense subset of $\mathbb{M}_{c}^{4}$ corresponding to $\mathbb{S} \mathbb{N}$ of $\mathbb{S}$, $\mathbb{N}:=\{s \in \mathbb{S} \mid$ at least one eigenvalue of $s=-1\}$.

\section{§ 4.2. The Lorentz Metric Compatible with the Group of Conformal Transformations on $\tilde{\mathbb{M}}, \hat{\mathbb{M}}_{c}^{4}$ and $\mathbb{M}^{4}$}

It was shown in our previous paper [6] that the metric

$$
g:=\left(d \eta^{0} \otimes d \eta^{0}+d \eta^{5} \otimes d \eta^{5}-d \eta^{p} \otimes d \eta^{p}\right) / r^{2}, \quad r^{2}:=\left(\left(\eta^{0}\right)^{2}+\left(\eta^{5}\right)^{2}\right),
$$

where $\eta^{\mu} d \eta_{\mu}=0$, is a pseudo-Riemannian metric on $\hat{\mathbb{M}}_{c}^{4}$ (and $\mathbb{M}_{c}^{4}$ as well). By using e.g. the parallelizable vector fields on $\hat{\mathbb{M}}_{c}^{4}$ (cf. [2] Proposition 7.3.2), it can easily be verified that the metric $g$ given in (4.3) is compatible with the group of conformal transformations ${ }^{4} \mathrm{SO}_{0}(2,4)$ on $\hat{\mathbb{M}}_{c}^{4}$, i.e., for $h \in \mathrm{SO}_{0}(2,4)$, we have

$$
\left.\left(h^{*} g^{\prime}\right)\right|_{[\eta]}=\left.\left(r / r^{\prime}\right)^{2} g\right|_{[\eta]}
$$

where $g^{\prime}:=\left.g\right|_{\left[\eta^{\prime}\right]},\left[\eta^{\prime}\right]=h[\eta] \in \hat{\mathbb{M}}_{c}^{4}$. With the aid of the differentiable covering map $p_{1}$, the uniquely determined lifted metric $\tilde{g}=p_{1}^{*} g$ on $\tilde{\mathbb{M}}$ is readily found:

$$
\tilde{g}=d \tau \otimes d \tau-d n^{p} \otimes d n^{p}, \quad n^{p} \cdot d n^{p}=0 .
$$

The transformation law given in (3.5) ensures that $\tilde{g}$ is compatible with $\overline{\mathrm{SU}(2,2)}$, i.e. we have an analog relation to (4.4) for $\tilde{g}$ :

$$
\left.\left(\tilde{h}^{*} \tilde{g}^{\prime}\right)\right|_{y_{1}}=\left.\left(r_{1} / r_{2}\right)^{2} \tilde{g}\right|_{y_{1}}, \quad \tilde{h} \in \overline{\mathrm{SU}(2,2)},
$$

\footnotetext{
4 See footnote of Eq. (4.9).
} 
where

$$
\tilde{g}^{\prime}=\left.\tilde{g}\right|_{y_{2}} ; \quad y_{1}, y_{2}=\tilde{h} y_{1} \in \tilde{\mathbb{M}}, \quad\left[\eta_{i}\right]=p_{1} y_{i}, \quad r_{i}^{2}=\left(\eta_{i}^{0}\right)^{2}+\left(\eta_{i}^{5}\right)^{2}, \quad i=1,2 .
$$

Equations (4.4) resp. (4.6) imply that the isometry subgroups of $\mathrm{SO}_{0}(2,4)$ resp. of $\overline{\mathrm{SU}}(2,2)$ on $\hat{\mathbb{M}}_{c}^{4}$ resp. on $\tilde{\mathbb{M}}$ are given by the seven-parameter $\mathrm{SO}_{0}(2) \times \mathrm{SO}_{0}(4)$ resp. $\mathbb{R} \times \operatorname{Spin}(4)$ (cf. [11] p. 347). The spaces therefore do not have the maximal possible ten parameter isometry group of a four-dimensional manifold. This is because they are not spaces of constant curvature, i.e., the metric does not fulfill the following equation

$$
R_{\mu v \xi \varrho}(x)=-K\left(g_{v \xi}(x) g_{\mu \varrho}(x)-g_{\mu \xi}(x) g_{v \varrho}(x)\right), \quad x \in M,
$$

where $R_{\mu v \xi \varrho}$ is Riemannian curvature tensor, $K=$ const. (cf. [24], $\S 13.7$ ).

If $f(y)$ is a positive $C^{\infty}$ function on $\tilde{\mathbb{M}}$, then $f(y) \tilde{g}$ is a metric which is conformally equivalent to $\tilde{g}$. The dimension of the isometry group of $f(y) \tilde{g}$ obviously depends on $f(y)^{5}$. We want to see how the number of isometries varies with $f$.

First of all we remark that we mainly want to study a $\overline{\mathrm{SU}(2,2)}$-invariant theory on the whole $\tilde{\mathbb{M}}$, and therefore the positive $C^{\infty}$ function $f$ should also well-defined everywhere on $\tilde{\mathbb{M}}$. This excludes, for instance, the case of an Einstein-de Sitter space which is conformally equivalent to a proper subset of $(\tilde{\mathbb{M}}, \tilde{g})$, because in this example the corresponding function $f$ is not well-defined everywhere on $\tilde{\mathbb{M}}$. (cf. [9] $§ 5.1-\S 5.3$, and [24] $\S 16.3$.) We remind also that $f$ must clearly be a homogeneous function of degree zero in the $\eta$-variables, cf. (4.3).

Having $f$ well-defined on the whole $\tilde{\mathbb{M}}$, Eq. (4.6) then implys

$$
\left(\tilde{h}^{*} f\left(y^{\prime}\right) \tilde{g}^{\prime}\right)_{y}=\frac{f\left(y^{\prime}\right)}{f(y)} \cdot \frac{r^{2}}{r^{\prime 2}}\left(f(y) \tilde{g}_{\mid y}\right), \quad \tilde{h} \in \overline{\mathrm{SU}(2,2)} .
$$

Equation (4.7) implys that the number of isometries of the metric $f(y) \tilde{g}$ cannot exceed the number of isometries of $\tilde{g}$, i.e., seven. (In using Eq. (4.6), one occasionally notices that the 15-parameter $\overline{\mathrm{SU}(2,2)}$ constitutes the conformal Lie transformation group of maximal dimension with respect to the 4-dimensional Lorentz manifold ( $\tilde{\mathbb{M}}, \tilde{g})$, cf. [13], p. 143, Theorem 6.1.)

To be physically meaningful, one still has to impose another conditions on the set $\{f\}$, this shall be done in $\S 6.3$.

The well-known Minkowski metric

$$
' g=d x^{0} \otimes d x^{0}-d x^{i} \otimes d x^{i}
$$

can be obtained via the local diffeomorphism

$$
\varphi_{\varkappa}^{-1}: \mathbb{M}^{4} \approx \varphi_{\varkappa} U_{\varkappa} \rightarrow U_{\varkappa} \subset \mathbb{M}_{c}^{4}
$$

from the conformal metric $g$ given in (4.3) in a suitable local coordinate $\varphi_{x}:[\eta]$ $\mapsto\left(x^{0}, \ldots, x^{3}\right) \in \mathbb{M}^{4}$ relative to appropriate basis vector fields in $T \mathbb{M}^{4}$. The group of isometries is the ten-parameter inhomogeneous Poincaré group $\mathrm{IO}_{0}(1,3)$, while the automorphisms of the $\mathrm{CO}(1,3)$-structure on $\mathbb{M}^{4}$, which is in 1-1-correspondence with ' $g$ (cf. [13] p. 9 Excercise 2.6), is obviously the eleven-parameter

\footnotetext{
5 The author is indebted to R. Haag and J. E. Roberts for reminding him of this important point.
} 
group $\mathrm{IO}_{0}(1,3) \otimes D_{1}, D_{1}$ being the 1-parameter dilatation. Since the so-called special conformal transformation $c$ given by

$$
\begin{gathered}
c: x^{\mu} \in \mathbb{M}^{4} \mapsto x^{\prime \mu}:=\frac{x^{\mu}+c^{\mu} x \cdot x}{\sigma(x ; c)}, \quad x \cdot x:=\left(x^{0}\right)^{2}-\sum_{i=1}^{3}\left(x^{i}\right)^{2} \\
\sigma(x ; c):=1+2 c \cdot x+(c \cdot c)(x \cdot x)
\end{gathered}
$$

is not a diffeomorphism of $\mathbb{M}^{4}$ onto itself, the group of special conformal transformation on $\mathbb{M}^{4}$ does not exist. $c$ can only be considered as a conformal mapping ${ }^{6}$ on $\mathbb{M}^{4}$, since the relation

$$
\left.\left(c^{* \prime} g_{m^{\prime}}\right)\right|_{m}=\left.(\sigma)^{-2 \prime} g\right|_{m}, \quad m \in \mathbb{M}^{4}
$$

formally holds, where ' $g_{m^{\prime}}=\left.{ }^{\prime} g\right|_{m^{\prime}}$ if $m^{\prime}=c(m) \in \mathbb{M}^{4}$.

\section{$\S$ 5. The Conformally Invariant Causality Property of ( $\tilde{\mathbb{M}}, \tilde{g})$}

\subsection{Some Concepts about Causality on a Lorentz Manifold and the Stable Causality of $(\tilde{\mathbb{M}}, \tilde{g})$}

It is well-known (cf. [9] and the literatures cited there) that a local causal structure can be defined on a four-dimensional connected Hausdorff differentiable manifold $M$ by assuming.

i) there is a Lorentz metric (at least of class $C^{2}$ ) on $M$, and

ii) $M$ is time-orientable.

(For precise definition of time-, space-, and topological orientality of a connected pseudo-Riemannian manifold $(M, g)$, cf. [31] p. 341.)

For a four-dimensional space-time $M$, the Lorentz metric $g$ of signature $(3,1)$, i.e. $(-,-,-,+)$, gives rise to some extra structure on $M$. The tangent vector $X_{m} \neq 0$ at a point $m \in M$ can be divided into three classes: $X_{m}$ is called timelike, lightlike or spacelike if $g_{m}\left(X_{m}, X_{m}\right)>0,=0$, or $<0$ respectively. The timeorientability of $M$ then enables us to decompose the subset $P$ of timelike tangent vectors of $T_{m} M$ into two separated sets: Those labelled by "future-directed" $\left(P_{+}\right)$ and those by "past-directed" $\left(P_{-}\right), P=P_{+} \cup P_{-}$. In a similar way, we say that a piecewise smooth curve $k: I \subset \mathbb{R} \rightarrow M$ is timelike, null or spacelike curve, if its tangent vectors $k^{\prime}(t)$ are timelike $\left(k^{\prime}(t) \in P\right)$, null $\left(k^{\prime}(t) \in \partial \bar{P}\right)$ or spacelike $\left(k^{\prime}(t)\right.$ $\in T_{k(t)}(\bar{P})$ for all $t \in I$. A curve $k$ is called causal iff it is timelike or null curve. On a Lorentz manifold $M$, a point $p \in M$ is said to be in the past (resp. strictly in the past) of another point $q \in M$, denoted by $p<q$ (resp. $p \ll q$ ), if $p$ can be connected to $q$ by a future-directed causal curve (resp. by a future-directed timelike curve) $k: \mathbb{R} \supset[0,1] \rightarrow M$ with $k(0)=p, k(1)=q$.

One of the properties of the metric thus ensures that the time labelling can be carried out in a continuous way locally. (The reason for the (at least) $C^{2}$-property of the metric is the field equation it has to satisfy in general relativity. This however

${ }^{6}$ Cf. [13] p. 9 Excercise 2.6, [31] p. 79 and [22]. A differentiable map $h:(M, g) \rightarrow\left(M^{\prime}, g^{\prime}\right)$ of pseudo-Riemannian manifolds is called conformal mapping, if there is a function $\lambda$ on $M$ such that $g_{h(x)}^{\prime}\left(h_{*} X, h_{*} Y\right)=\lambda(x) g_{x}(X, Y)$, for all $x \in M ; X, Y \in T_{x} M$. If the inverse map $h^{-1}$ exists, i.e. if $\lambda(x)$ never vanishes, and is also conformal, then $h$ is called a conformal diffeomorphism. If $h$ is a conformal diffeomorphism of $(M, g)$ onto itself, then $h$ is called a conformal transformation of $(M, g)$. 
shall not be treated here.) But whether the time labelling can be carried out continuously over the whole manifold depends on the global connectivity property in relation to the null cones. In this connection, it seems also physically reasonable to demand that $M$ shall not admit closed timelike curve. This forced us for instance to abadon a compact Four-Lorentz manifold as a possible domain of a physical theory, since it is known that a compact Four-Lorentz manifold admits a Lorentz metric iff its Euler-Poincaré characteristic vanishes (cf. e.g. [29] p. 207). Even if this is the case, every such compact Four-Lorentz manifold has closed timelike curve $[1,4]$.

We call a non-compact Four-Lorentz manifold causal, if it contains no closed causal curve. It turns out that the strongest causality property is the so-called stable causality: A non-compact manifold $M$ is stably causal, if there exists an open set of metrics (in $C^{0}$-topology as section of $T^{*} M \otimes T^{*} M$ ) which are causal and contains the given metric.

To see if a non-compact Four-Lorentz manifold is stably causal, we quote a theorem due to Hawking [10]: A non-compact Four-Lorentz manifold is stably causal iff it has a time function. By definition (cf. [31] p. 339) a time function on an open subset $U$ of a Lorentz manifold $M$ is a smooth real-valued function $f$ such that $g(\operatorname{grad} f, \operatorname{grad} f)=+1$, if the Lorentz metric has the signature $(3,1)$.

It is easy to see that the space $(\tilde{\mathbb{M}}, \tilde{g})$ is time-, space-, and topologically orientable, since the linear holonomy group of the simply-connected $(\tilde{\mathbb{M}}, \tilde{g})$ is contained in the isometry group $\widehat{\mathrm{SO}_{0}(2) \times \mathrm{SO}_{0}(4)}$ of $\tilde{g}$ (cf. [31] §3.4).

Since $f(y):=\tau$ is a time function on $\widetilde{\mathbb{M}}$ relative to the metric $\tilde{g}$ given in (4.5), $(\tilde{\mathbb{M}}, \tilde{g})$ is therefore stably causal.

\subsection{The Conformal Invariant Causal Structure on ( $\tilde{\mathbb{M}}, \tilde{g})$}

From the last section it is clear how to define the causal relationship between the points of $\tilde{\mathbb{M}}$. The future-directed (resp. past-directed) tangent vector

$$
Y_{y}=b^{0} \frac{\partial}{\partial \tau}+b^{p} \frac{\partial}{\partial n^{p}} \in T_{y} \tilde{\mathbb{M}}, \quad b^{p} \cdot n^{p}=0
$$

at a point $y \in \tilde{\mathbb{M}}$ is defined by $b^{0}>0\left(\right.$ resp. $\left.b^{0}<0\right) . Y_{y}$ is timelike, lightlike or spacelike according to $\tilde{g}_{y}\left(Y_{y}, Y_{y}\right)>0,=0$, or $<0$ respectively.

To obtain a conformal invariant causal ordering, it is clear that

i) the division of tangent space in three parts and

ii) the "future-directed" (resp. "past-directed") property of a timelike or a lightlike tangent vector must remain invariant under the action of conformal transformations given in (3.5).

The first point is clear following (4.6). The second point amounts to showing that for $\tilde{h} \in \overline{\mathrm{SU}(2,2)}: y_{1} \mapsto y_{2}, \quad \tilde{h}_{*} Y_{1}=Y_{2}$ with $Y_{i}=\left(b_{i}^{0} \partial / \partial \tau+b_{i}^{p} \partial / \partial n^{p}\right)_{y_{i}} \in T_{y_{i}} \mathbb{\mathbb { M }}$, the equality

$$
\operatorname{sgn}\left(b_{1}^{0}\right)=\operatorname{sgn}\left(b_{2}^{0}\right)
$$

holds, where $b_{2}^{0}=\left(b_{1}^{0}(1+\partial / \partial \tau)+b_{1}^{p} \partial / \partial n^{p}\right) f\left(\zeta_{\tilde{h}}\right), \tilde{h} \in \overline{\mathrm{SU}(2,2)}$. But since the transformation law (3.5) implys that the derived mapping $p_{1 *}$ is compatible with the action of $\overline{\mathrm{SU}(2,2)}$ on $T \overline{\mathbb{M}}$ and the action of $\mathrm{SU}(2,2)$ on $T \hat{\mathbb{M}}_{c}^{4}$, we get

$$
\operatorname{sgn}\left(b_{i}^{0}\right)=\operatorname{sgn}\left(a_{i}^{0} \eta_{i}^{5}-a_{i}^{5} \eta_{i}^{0}\right), \quad i=1,2,
$$


where $p_{1 *} Y_{i}=\left[\left(p_{1}\left(y_{i}\right), a_{i}^{u} \partial / \partial \eta^{\mu}\right)\right] \in T_{p_{1}\left(y_{i}\right)} \hat{\mathbb{M}}_{c}^{4}$. (Notice that $p_{1 *} \partial /\left.\partial \tau\right|_{y}=\left[\left(p_{1}\left(y_{i}\right)\right.\right.$, $\left.\left.\eta^{5} \partial / \partial \eta^{0}-\eta^{0} \partial / \partial \eta^{5}\right)\right] \in T_{[\eta]}\left(\hat{\mathbb{M}}_{c}^{4}\right),[\eta]=p_{1}(y)$. We remind also that [7]

iff

$$
\left(\eta^{\prime}, a^{\prime \mu} \partial_{\mu}^{\prime}\right), \quad\left(\eta, a^{\mu} \partial_{\mu}\right) \in\left[\left(\eta, a^{\mu} \partial_{\mu}\right)\right] \in T_{[\eta]}\left(\hat{\mathbb{M}}_{c}^{4}\right)
$$

1) $\eta^{\prime}=\varrho \eta ; a^{\prime}=\varrho a+\delta \eta$ for $\varrho \in \mathbb{R}_{+}$and $\delta \in \mathbb{R}$;

2) $b_{4}^{6}(a, \eta)=0$.

On the other hand, we proved in our previous paper [7] that given $\left[\left(\eta, a^{\mu} \partial / \partial \eta^{\mu}\right)\right] \in T_{[\eta]}\left(\mathbb{M}_{c}^{4}\right)$ then the $\operatorname{sgn}\left(a^{5} \eta^{0}-a^{0} \eta^{5}\right)$ is invariant under the action of $0^{\dagger}(2,4)$ iff $g_{[\eta]}\left(a^{\mu} \partial_{\mu}, a^{\mu} \partial_{\mu}\right) \geqq 0$ with respect to $g$ given in (4.3). The second point is thus confirmed.

\section{§ 6. Some Remarks}

\subsection{Why Does the Special Conformal Transformation in $\mathrm{M}^{4}$ "Destroy" Causality?}

Compared with the general causality concept on a Four-Lorentz manifold as described in $\S 5.1$, the usual well-known causality concept in Minkowski space $\mathbb{M}^{4}$ (" $\mathbb{M}^{4}$-causality") turns out to be a special case: one only has to take the straight line $l_{x y}$ in $\mathbb{M}^{4}$ joining the two points $x, y \in \mathbb{M}^{4}$ under consideration to determine their respective $\mathbb{M}^{4}$-causality relation according to the timelike, lightlike or spacelike character of this straight line (i.e., according to $b_{3}^{4}(x-y, x-y)>0$, $=0$, or $<0$ respectively) as well as the $\operatorname{sgn}(x-y)$. As the Poincaré group $\mathscr{P}$ acting as Lie transformation group on $\mathbb{M}^{4}$ leaves the straight line and the symmetric bilinear form $b_{3}^{4}$ invariant, while $\operatorname{sgn}(x-y)$ is invariant under the orthochronous Poincaré group $\mathscr{P}^{\uparrow}, \mathbb{M}^{4}$-causality is invariant under $\mathscr{P}^{\dagger}$ (cf. [33]). The $\mathbb{M}^{4}$-causal relationship for the transformed $x^{\prime}=h x, y^{\prime}=h y, h \in \mathscr{P}^{\dagger}$, is determined thereby again by a straight line $l_{x^{\prime} y^{\prime}}$ lying entirely in $\mathbb{M}^{4}$.

What is changing, if one considers the conformal mapping $c$ as given in (4.9) on $\mathbb{M}^{4}$ ?

A straight line will be mapped into a curved line but preserving the angle between two curves. This last property ensures the invariance of the division of tangent vectors of tangent space into 3 classes [cf. (4.10), assuming $\sigma \neq 0$ for a moment]. And as we have shown in [7], the division of timelike and lightlike tangent vectors into two future- and past-directed parts is also invariant under $c$. Therefore, in this sense, $c$ does respect the causality locally. In fact, if we restrict ourself to an open set $U(0 ; c)$ defined by

$$
U(0 ; c):=\left\{y \in \mathbb{M}^{4} \mid \sigma(y ; c)>0\right\},
$$

then the conformal mapping $c$ would not destroy the causality relation of $y \in U(0 ; c)$ relative to 0 after the mapping even in $\mathbb{M}^{4}$-causality. But for all points $y^{\prime} \in U^{\prime}(0 ; c)$,

$$
U^{\prime}(0 ; c):=\complement \overline{U(0 ; c)} \cap L_{0},
$$

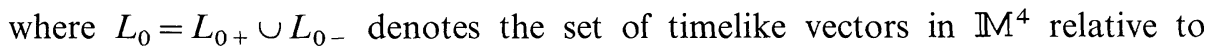
$0 \in \mathbb{M}^{4}$, the special conformal mapping $c$ destroys the $\mathbb{M}^{4}$-causality. 
Is it however justified to use $\mathbb{M}^{4}$-causality to judge if $c 0$ and $c y^{\prime}$ still have their old causality relation to each other? We do not think so, since $c$ is singular on $\partial \bar{U}$. Therefore, the straight line $l_{0 y}$ joining 0 and $y^{\prime} \in U^{\prime}(0 ; c)$ would be transformed into three disjoint parts of curves as viewed from $\mathbb{M}^{4}$. (This is the main difference of $c$ compared to $\mathscr{P}^{\uparrow} \otimes D_{1}$ !) These three parts are: $l_{0 y^{\prime}} \cap U$ would be transformed into a curved line in

$$
I^{+}(c 0):=\left\{z \in \mathbb{M}^{4} \mid z \gg c 0\right\} \quad \text { or } \quad I^{-}(c 0):=\left\{z \in \mathbb{M}^{4} \mid z \ll c 0\right\}
$$

according to $\operatorname{sgn}\left(y^{\prime 0}\right)>0$ or $<0$ before the mapping, respectively; $l_{0 y^{\prime}} \cap U^{\prime}$ would be transformed into a curved line in $\left.\mathbb{M}^{4} \backslash \overline{\left\{I^{+}(c 0) \cup I^{-}(c 0)\right.}\right\}$; while the zero-curve (i.e. curve containing only one point) $l_{0 y^{\prime}} \cap \partial \bar{U}$ into "infinities" of $\mathbb{M}^{4}$, i.e., after the $c$-mapping, this zero curve does not belong to $\mathbb{M}^{4}$ anymore. The following figures for $l_{0 y^{\prime}} \cap L_{0+} \neq \emptyset$ shall illustrate what we mentioned above (by supposing $c \cdot c \neq 0)$ :

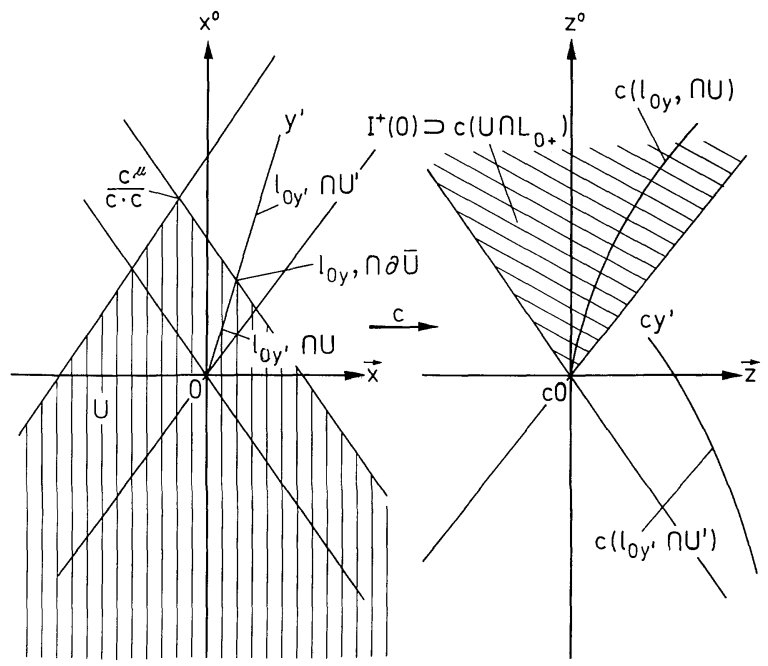

\subsection{Some Remarks on the Infinitesimal Conformal Transformation on $\mathbb{M}^{4}$}

To avoid the "causality-violating" property of the conformal transformation on $\mathbb{M}^{4}$, some people consider the so-called "infinitesimal conformal transformation" $\underline{c}$, induced by the element of the special conformal group acting on $\mathbb{M}^{4}$,

$$
\underline{c}: x^{\mu} \mapsto x^{\prime \mu}:=(1-2 \underline{c} \cdot x) x^{\mu}+\underline{c}^{\mu} x \cdot x .
$$

This implies

$$
\left(\left(\underline{c}^{*} g^{\prime}\right)_{x}\right)_{\mu \nu}=\left.(1-4 \underline{c} \cdot x) g_{\mu \nu}\right|_{x}+O\left(x ; \underline{c}^{2}\right)_{\mu \nu} .
$$

To avoid for instance the vanishing of $1-4 \underline{c} \cdot x$, that would obviously destroy any conformal character of the mapping $\underline{c}$, one is forced to consider the mapping 
$\underline{c}$ acting only on an open neighborhood of $x, U_{\underline{c}}(x) \subset \mathbb{M}^{4}$, such that $O\left(x ; \underline{c}^{2}\right)$ can be neglected compared with the factor $1-4 \underline{c} \cdot x \neq 0$.

What kind of transformation is it?

Each such 1-parameter transformation has to be understood as a local 1parameter group of local diffeomorphism at $z \in \mathbb{M}$. (cf. [31] p. 4). It consists of a neighborhood $V_{\varepsilon}(z)$ of $z$, a constant $\varepsilon>0$, and a family $\left\{\phi_{t}\right\}_{|t|<\varepsilon}$ such that

i) $\phi_{t}$ is a diffeomorphism of $V_{\varepsilon}(z)$ onto $\phi_{t}(V) \subset \mathbb{M}$;

ii) the map $(-\varepsilon, \varepsilon) \times V \rightarrow \mathbb{M}$ given by $(t, x) \mapsto \phi_{t}(x)$ is differentiable; and (6.5)

iii) $\phi_{s}\left(\phi_{t}(x)\right)=\phi_{s+t}(x)$ whenever $x, \phi_{t}(x) \in V$ and $|s|,|t|,|s+t|<\varepsilon$.

It is now clear that the set of mappings $\underline{c}$ as given in (6.3) constitute a $\varepsilon$-local four-parameter conformal group of local diffeomorphisms at, say, $x \in \mathbb{M}^{4}$. The first "local" refers to group parameters $\left(\underline{c}^{\mu}\right)$, e.g. $\left(\Sigma_{\mu=1}^{4}\left(\underline{c}^{\mu}\right)^{2}\right)^{1 / 2}<\varepsilon$, the second "local" to the space-time points in an open neighborhood of $x, U_{\varepsilon}(x)$ (cf. also $[20] \S 5.9)$.

Any $\varepsilon$-local one-parameter conformal group $\phi_{t}$ induces a conformal vector field $X$ on $U_{\varepsilon}(x)$ by

$$
(X f)(y):=\left.\frac{d}{d t} f\left(\phi_{t}(y)\right)\right|_{t=0}, \quad f \in C^{\infty}(U), \quad y \in U_{\varepsilon}(x) .
$$

$X$ is the generator of the infinitesimal conformal transformation on $M$. It is known ([32] Chapter VII) that a vector field $X$ on $(M, g)$ is conformal iff

$$
\mathscr{L}_{X} g:=\lim _{t \rightarrow 0} \frac{1}{t}\left(g-\phi_{t}^{*} g\right)=\beta g
$$

for some function $\beta$ on $M$, where $\mathscr{L}_{X}$ is the Lie derivative relative to $X$.

The induced vector fields on $U_{\varepsilon}(x) \subset \mathbb{M}^{4}$ are

$$
\left.X_{\mu}\right|_{y}=(y \cdot y) \partial / \partial y^{\mu}-2 y_{\mu} y^{v} \partial / \partial y^{v}, \quad y \in U_{\varepsilon}(x), \quad \mu=0, \ldots, 3 .
$$

They fulfill (6.6) with $\beta=4 y$ for each $\left.X_{\mu}\right|_{y}$ and hence are local conformal vector fields, just like the mapping (4.9) induced by the elements of conformal group $c$ satisfies the condition explained e.g. in [31] p. 49 to be only a conformal mapping by virtue of (4.10). The point we want to emphasize here once again is: one should not forget the domain of definition $U_{\varepsilon}(x)$ of these local conformal vector fields [remember (6.4)!], if one tries to give any physical interpretation of the "infinitesimal conformal transformation" $\underline{c}$ on $\mathbb{M}^{4}$. However, if one agrees that we have a local four-parameter conformal group of local diffeomorphism, then the next question arises: what is the meaning of these kinds of operations, i.e. local diffeomorphisms, physically?

Of course, it is also possible to consider the $\mathrm{SO}_{0}(2,4)$-Lie-Algebra alone and forget the special conformal group as global or local transformation group on $\mathbb{M}^{4}$. In this case, there is the question: what is the meaning of the special conformal algebra being treated on the same footing as the Poincaré and dilatation algebra?

\subsection{Remarks on $(\tilde{\mathbb{M}}, \tilde{g})$}

The discussions in the last two sections showed in different ways that $\mathbb{M}^{4}$ is hardly to be a suitable domain to treat the group of conformal transformation by the same simple reason: the (infinitesimal) conformal mappings given in (4.9) 
and (6.3) are not diffeomorphisms of $\mathbb{M}^{4}$ onto $\mathbb{M}^{4}$. It is therefore natural to extend $\mathbb{M}^{4}$ into a compactified $\hat{M}_{c}^{4}$ (or $\mathbb{M}_{c}^{4}$, we shall not repeat the similar statement for $\mathbb{M}_{c}^{4}$ in the sequel). But following the remark in $\S 5.1$, the acausal $\hat{\mathbb{M}}_{c}^{4}$ (cf. also [7]) may not serve as reasonable domain for a physical theory. To investigate the possible physical relevance of the conformal group, the next logical step is to consider the universal covering space $\tilde{\mathbb{M}}$ of $\hat{\mathbb{M}}_{c}^{4}$ (cf. $[19,28,30]$ ), which has been shown in $\S 4$ and $\S 5$ to be a stable causal manifold admitting the conformal group $\widetilde{\mathrm{SU}(2,2)}$ acting on it as Lie transformation group which respects causality. From this standpoint, $\tilde{\mathbb{M}}$ is a physically acceptable non-compact FourLorentz manifold having the conformal group as its space-time transformation group, not only in infinitesimal sense but also in a global manner.

The physical relevance of the conformal group as transformation group shall therefore be interpreted with respect to the space $\tilde{\mathbb{M}}$ but not $\mathbb{M}^{4}$ as attempted in $[12,15]$. We shall not try in this paper to give any detailed interpretation of conformal transformation other than the obvious "rescalling" property exhibited in (4.4) and (4.6), that is in the same spirit as the interpretation given by Kastrup [12]. However, following the discussion in $\S 4.2$, there are only at most seven isometric vector fields (Killing vector fields) on $(\tilde{\mathbb{M}}, f \tilde{g})$, where the maximal number of isometries will only be assumed by $f=$ const $>0$.

What is the meaning of these isometric vector fields?

It is well-known that in a Lagrangian field theory a definite and unique formula ${ }^{7}$ for the symmetric energy-stress tensor $T^{\mu v}$ can be derived by varying the action with respect to the metric, while in general relativity the energy-stress tensor can be considered as uniquely defined by the "other" side of Einstein field equation. The first physical restriction to be imposed on the set of $\{f\}$ is therefore the following one: The energy-stress tensor corresponding to each metric $f \tilde{g}$ should be physically reasonable. Furthermore, the commonly postulated zero-divergence condition on $T^{\mu v}, T^{\mu v} ;{ }_{v}=0$, imposes the second restriction on $f$. Supposed that the set of such $f$ is not void (otherwise no reasonable physical theory can be formulated). Since it is well-known (cf. e.g. [24] § 13.6) that the existence of Killing vector field $X_{v}$ is equivalent to the existence of the conserved vector field $P^{\mu}=T^{\mu v} X_{v}$, by taking $f=1$ in $f \tilde{g}$, we would get a maximal set of conserved vector fields $P^{\mu}$ whose corresponding Killing vector fields $X_{v}$ may be related to the generators of energy and rotations in $S^{3}$, as the isometry group of $\tilde{g}$ is $\overline{\mathrm{SO}_{0}(2) \times \mathrm{SO}_{0}(4)} \approx \mathbb{R}$ $\times$ Spin(4). These seven Killing vector fields on $T \tilde{\mathbb{M}}$ are

$$
\begin{gathered}
-i \tilde{L}_{50}:=\partial / \partial \tau \\
\left(\tilde{Q}_{k}\right):=-i\left(\tilde{L}_{23}, \tilde{L}_{31}, \tilde{L}_{12}\right), \quad\left(\tilde{N}_{k}\right):=-i\left(\tilde{L}_{14}, \tilde{L}_{24}, \tilde{L}_{34}\right)
\end{gathered}
$$

where $\tilde{L}_{j k}=i\left(n_{j} \cdot \partial / \partial n^{k}-n_{k} \cdot \partial / \partial n^{j}\right), 1 \leqq j, k \leqq 4$. Their image under $p_{1 *}$ on $T \hat{\mathbb{M}}_{c}^{4}$ are

$$
\begin{aligned}
p_{1 *} \tilde{L}_{50} & =\hat{L}_{50}=\frac{1}{2}\left(\hat{P}_{0}+\hat{K}_{0}\right) \\
p_{1 *}\left(i \tilde{Q}_{k}\right) & =\left(\hat{L}_{23}, \hat{L}_{31}, \hat{L}_{12}\right) \\
p_{1 *}\left(i \tilde{N}_{j}\right) & =\left(\hat{L}_{14}, \hat{L}_{24}, \hat{L}_{34}\right)=-\frac{1}{2}\left(\hat{P}_{j}-\hat{K}_{j}\right)
\end{aligned}
$$

\footnotetext{
${ }^{7}$ In the case that the equations of the fields can be derived from the Lagrangian.
} 
where $\hat{L}_{\mu \nu}:=i\left(\eta_{\mu} \partial_{\nu}-\eta_{\nu} \partial_{\mu}\right), \partial_{\nu}:=\partial / \partial \eta^{\nu}, 0 \leqq \mu, v \leqq 5$ are generators of "rotations" in $(\mu, v)$-plane of $\hat{\mathbb{M}}_{c}^{4}$;

$$
\hat{P}_{\mu}=\hat{L}_{5 \mu}+\hat{L}_{4 \mu}, \quad \hat{K}_{\mu}=\hat{L}_{5 \mu}-\hat{L}_{4 \mu} \quad 0 \leqq \mu \leqq 3
$$

are generators of translations and special conformal transformations in $\hat{M}_{c}^{4}$ (cf. [18]).

$\tilde{L}_{50}$ can obviously be regarded as the "total energy operator" for a conformal invariant theory on $\tilde{\mathbb{M}}$ (cf. [16]). It then follows that $\left(\hat{P}_{0}+\hat{K}_{0}\right) / 2$ plays the same role for a conformal invariant theory on $\hat{\mathbb{M}}_{c}^{4}$.

$\tilde{Q}_{k}$ and $\tilde{N}_{k}$ generate six rotations in the space part $S^{3}$ of $\tilde{\mathbb{M}}$. As we identified the Minkowski space $\mathbb{M}^{4}$ with an open dense subset $U_{\varkappa}$ or $\hat{\mathbb{M}}_{c}^{4}$, where $x^{\mu}=\eta^{\mu} / x$, $\mu=0,1,2,3$ and $x=\eta^{4}+\eta^{5} \neq 0, \tilde{Q}_{k}$ correspond to the pure space rotations in the space part $\mathbb{R}^{3}$ of $\mathbb{M}^{4} . \tilde{Q}_{k}$ can therefore be interpreted as generators of conserved "angular momentum" in $\tilde{\mathbb{M}}$. On the other hand, the "boost" along the $j$-axis in $\mathbb{M}^{4}$ is replaced by $i \tilde{N}_{j} \sim-\left(\hat{P}_{j}-\hat{K}_{j}\right) / 2 . i \tilde{N}_{j}$ are, as viewed from $\mathbb{M}^{4}$, generators of transformations responsible for the mixing of finite part and "infinities" of $\mathbb{M}^{4}$. In a naive manner, we would try to interprete them as generators of conserved "extended angular momentum" in $\tilde{\mathbb{M}}$.

The absence of three Killing vector fields in $\tilde{\mathbb{M}}$ may have something to do with the non-conservation of "linear momentum" in $\tilde{\mathbb{M}}$ in a global manner. These quantities can still have an approximate conservation law in a small region of $\tilde{\mathbb{M}}$. However, it is not clear how this approximate conservation law behaves with respect to the different "small regions" of $\tilde{\mathbb{M}}$.

Acknowledgement. I am indebted to Prof. H. A. Kastrup for critical comments and many valuable discussions. I thank Dr. D. H. Mayer for a critical comment. By this opportunity I also want to express my sincere gratitude to Prof. R. Haag for his constructive critics and helps, that bring this paper into a more clear and concise presentation. It is my pleasures to thank Drs. Peter und Susanne Dierolf from Mathematisches Institut der Universität München for a private communication.

Finally, I owe the Bundesministerium für Forschung und Technologie for financial support, without it this work could not be done.

\section{References}

1. Bass, R. W., Witten, L.: Rev. Mod. Phys. 29, 452 (1957)

2. Brickell, F., Clark, R.S.: Differential Manifolds. London: Van Nostrand Reinhold Co. 1970

3. Dierolf, P., Dierolf, S.: Private communication

4. Geroch, R.: J. Math. Phys. 8, 782 (1967)

5. Glaser, V.: Commun. math. Phys. 37, 257-272 (1974)

6. Go.T.H., Mayer, D.: Rep. Math. Phys. 5, 187-202 (1974)

7. Go,T.H., Kastrup, H.A., Mayer, D.: Properties of Dilatations and Conformal Transformations in Minkowski space, rev. version TH Aachen Preprint 1973

8. Graev, M.I.: Amer. Math. Soc. Transl. (2) 66, 1-62 (1968), § 1

9. Hawking, S. W., Ellis, G.F.R.: The Large Scale Structure of Space-Time. London: Camb. Uni. Press 1973

10. Hawking, S. W.: Proc. Roy. Soc. London, ser A 308, 433-436 (1969)

11. Helgason, S.: Diff. Geom. and Symmetry Spaces. New York: Academic Press 1962

${ }^{8}$ For example, in analogy with the total energy operator $i \frac{\partial}{\partial \tau}$ of $\tilde{\mathbb{M}}$, we may consider $p_{1 *}^{-1}\left(\frac{1}{2}\right.$ $\left.\left(\hat{P}_{J}+\hat{K}_{j}\right)\right)=i\left(\cos \tau \cdot \partial / \partial n^{j}+n_{J} \sin \tau \cdot \partial / \partial \tau\right)$ as "linear momentum operator" in $\tilde{\mathbb{M}}$. 
12. Kastrup,H. A.: Ann. Phys. 9, 388 (1962); Nucl. Phy. 58 (1964)

13. Kobayashi, S.: Transformation Groups in Diff. Geom. Berlin-Heidelberg-New York: Springer 1972

14. Kobayashi, S., Nomizu,K.: Foundations of Diff. Geom. I. New York: Interscience Publ. 1963

15. Laue, H.: Nuovo Cimento 3 B, 55 (1971)

16. Lüscher, M., Mack, G.: Global Conformal Invariance in QFT Preprint of Uni. Bern, August 1974

17. Mack, G.: Group Theor. Approach to Conf. Inv. QFT, Preprint of Uni. Bern, February, 1974

18. Mack, G., Salam, A.: Ann. Phys. (N.Y.) 53, 174 (1969)

19. Mayer,D.: Conf. Invariant Causal Structures on Pseudo-Riemannian manifolds, Preprint of TH Aachen, April, 1974

20. Miller, W.: Symmetry groups and their applications. New York: Academic Press 1972

21. Osterwalder, K., Schrader, R.: Commun. Math. Phys. 31, 83-112 (1973)

22. Obata, M.: J. Diff. Geom. 4, 311-333 (1970)

23. Penrose, R.: Structure of Space-Time. In: Battle Rencontres, ed. C. DeWitt and J. A. Wheeler. New York: W. A. Benjamin Inc. 1968

24. Robertson, H. P., Noonan, T.W.: Relativity and Cosmology, Philadelphia: W. B. Sanders Comp. 1969

25. Rühl,W.: Commun. math. Phys. 27, 53-86 (1972); Conformal Kinematic, Preprint of Uni. Kaiserslautern, 1973

26. Schaaf, M.: Reports on Math. Physics, 4, 275-279, 1973

27. Schroer, B., Swieca, J.A.: Phys. Rev. D, Vol. 10, 480 (1974)

28. Segal,I.E.: Covariant Chronogeometry and Extragalactic Astronomy, MIT-Preprint, 1973

29. Steenrod, N.: The Topology of Fibre Bundles, Princeton: University Press, 1951

30. Todorov,I. T.: CERN-Preprint, Ref. TH. 1697-CERN, 1973

31. Wolf, J. A.: Spaces of Constant Curvatures. New York: McGraw-Hill 1967

32. Yano, K.: The theory of lie derivative and its applications. Amsterdam: North. Holl. 1957

33. Zeemann,E. C.: J. Math. Phys. 5, 490-493 (1964), cf. also [1] [4] [9] [23]

Communicated by R. Haag

Tjoe-hian Go

Institut für Theoretische Physik

der Universität Bern

Sidlerstraße 5

CH-3012 Bern, Switzerland 
